Les ANNALES Les Annales de droit

DE DROIT

$8 \mid 2014$

Varia

\title{
La question juridique des restes humains sous l'angle de la dignité de la personne
}

The legal issue of human remains from the perspective of human dignity

\section{Guillaume Fontanieu}

\section{(2) OpenEdition \\ 12 Journals}

Édition électronique

URL : http://journals.openedition.org/add/739

DOI : $10.4000 /$ add. 739

ISSN : 2606-1988

Éditeur

Presses universitaires de Rouen et du Havre

\section{Édition imprimée}

Date de publication : 1 mai 2014

Pagination : 197-227

ISBN : 979-10-240-0161-6

ISSN : 1955-0855

Référence électronique

Guillaume Fontanieu, «La question juridique des restes humains sous l'angle de la dignité de la personne », Les Annales de droit [En ligne], 8 | 2014, mis en ligne le 14 mars 2018, consulté le 17 septembre 2019. URL : http://journals.openedition.org/add/739 ; DOI : 10.4000/add.739 
- la théorie de Kelsen, tout comme sa métathéorie, est prescriptive;

- si l'on s'en tient à la distinction humienne, principe au cœur du construit (néo)normativiste, alors le (néo)normativiste n’a pas pour objet le droit, puisque seul le droit valide constitue, selon les thèses afférentes, du droit ;

si l'on accepte la démonstration de la validité du droit par Kelsen, il faut accepter, parallèlement et obligatoirement, le syncrétisme inévitable des méthodes normative et causale;

- par conséquent, le (néo)normativisme n'est pas la seule méthode proprement normative (ou juridique), puisqu'il ne peut démontrer l'existence du droit sans recourir au fait.

Au regard de ces conclusions, le lecteur attentif opérera inévitablement un choix parmi les quatre options suivantes:

1. Considérer qu'il n'y a pas de choix à effectuer (mais c'est encore un choix !)

2. Considérer que les démonstrations présentes ne valent pas. Il n'y a pas, en effet, en science, de vérité absolue.

3. Considérer que le normativisme est un échec, en ce qu'il a succombé à l'échec de l'idée d'inférence et ne peut, selon ses propres thèses, démontrer l'existence d'un droit positif. Il faut alors recourir à une autre structure conceptuelle unitaire entièrement nouvelle. Ce que certaines théories, notamment la théorie réaliste de l'interprétation de Michel Troper, semblent proposer. L'on peine, toutefois, à admettre la logique du projet d'une science qui se dit distincte de son objet et qui prétend que toute science constitue son objet, opérant une conciliation entre les deux pôles extrêmes des épistémologies positivistes et constructivistes.

4. Admettre que le constructivisme de Kelsen et de ses épigones est une étape importante, non négligeable, et que la science se construit par la succession de ces étapes. Il faut alors, dans une démarche dialectique - tant avec l'objet qu'avec l'héritage scientifique -, faire le deuil d'un constructivisme radical basé sur l'unité pour, enfin, admettre que le droit est complexité.

Doctorant contractuel en droit constitutionnel université de Rouen 



\title{
La question juridique des restes humains sous l'angle de la dignité de la personne
}

\author{
Guillaume FonTANIEU
}

Les morts n'ont plus que les vivants pour ressource ${ }^{1}$.

Les pratiques culturelles, rituelles, de peuples anciennement colonisés et demandant aujourd'hui réparation remettent en perspective nos traditions et objets juridiques. Une des requêtes mettant en évidence cet écart conceptuel s'est formalisée autour des restes humains contenus dans les collections publiques et issus de ces anciennes colonies. La mort est un objet juridique singulier pour lequel de nombreux détours furent empruntés pour en formaliser un statut. Comme l'écrivait le doyen Carbonnier, la mort «est l'évanouissement de la personnalité [...] cette vérité est si affreuse que l'on s'efforce de la dissimuler ${ }^{2}$ ». En effet, il a longtemps été admis que la France faisait mourir par décret ${ }^{3}$, jusqu'à ce que la loi $^{4}$ du 19 décembre 2008 vienne mettre en forme les pratiques funéraires. Ainsi, le statut juridique d'un reste humain est en France, suffisamment clarifiée: la perte de la personnalité juridique du fait sa condition d'inanimé en fait une chose, mais sa qualité d'appartenance

1. Paul Valéry, "Discours de réception à l'Académie française ", prononcé le 23 juin 1927, disponible en ligne à l’adresse http://www.academie-francaise.fr/discours-de-reception-et-reponse-de-m-gabriel-hanotaux (consulté le 27 janvier 2013).

2. Jean Carbonnier, Droit civil, $12^{\mathrm{e}}$ éd., Paris, PUF, t. I, 1979, p. 227.

3. Décret $n^{\circ} 41-5050$ du 31 décembre 1941 codifiant les textes relatifs aux opérations d'inhumation, d'exhumation, d'incinération et de transport des corps.

4. Loi $\mathrm{n}^{0}$ 2008-1350 du 19 décembre 2008 relative à la législation funéraire, JORF $\mathrm{n}^{\mathrm{O}} 0296$ du 20 décembre 2008, p. 19538, créant notamment, l’article 16-1-1 du Code civil. 
au genre humain le rend toutefois insusceptible d'appropriation ${ }^{5}$. Dans le cadre de la protection des restes humains, intervient un principe supérieur, celui de la dignité humaine permettant, même une fois l'affection familiale ${ }^{6}$ passée, d'estimer qu'ils «ne sont pas des biens comme les autres [...] par [ce] principe dans son double développement, dignité de la personne, dignité de l'humain ${ }^{7}$ ». Dès lors, les nombreuses collections de musées, détentrices ou gardiennes de restes humains, doivent prendre en considération cette variable quant à leur utilisation dans le cadre d'une exposition ou de travaux de recherche.

Si le principe juridique de la dignité humaine peut apparaître comme un "concept nouveau ${ }^{8}$ " au contenu imprécis et casuistique, il est reconnu désormais comme ayant une portée « universelle ${ }^{9}$ » et possède en France une valeur constitutionnelle ${ }^{10}$. En tant que composante de l'ordre public immatériel, la dignité humaine remet en question des pratiques, jusqu'alors institutionnalisées, comme la présence des restes humains dans des musées ou expositions publiques. Cette problématique a fait l'objet en 2010 d'un avis ${ }^{11}$ rendu par le Comité consultatif national d'éthique pour les sciences de la vie et de la santé ${ }^{12}$, faisant suite à la polémique suscitée en 2009 par l'exposition Our Body/À corps ouvert. Cette dernière, sous un prétexte scientifique et éducatif, présentait des

5. Article 16-1 du Code civil: "Chacun a droit au respect de son corps. Le corps humain est inviolable. Le corps humain, ses éléments et ses produits ne peuvent faire l'objet d'un droit patrimonial. »

6. TGI Lille, ord., 5 décembre 1996, Recueil Dalloz, 1997, p. 376: «La dépouille mortelle de l'individu fait l'objet d'un droit de copropriété familial, inviolable et sacré $[. .$.$] des raisons d'ordre public font que tout élément du corps humain en état$ de désagrégation, qui provient d'une sépulture fût-elle abandonnée, est digne de protection.»

7. Marie Cornu, "Le corps humain au musée, de la personne à la chose? », Recueil Dalloz, 2009, p. 1910.

8. Bernard Edelman, «La dignité humaine, un concept nouveau », Recueil Dalloz, 1997, p. $185-188$.

9. Article $1^{\text {er }}$, Déclaration universelle des droits de l'homme du 10 décembre 1948 : "Tous les êtres humains naissent libres et égaux en dignité et en droits ".

10. CC, décision $\mathrm{n}^{\circ} 94-343 / 344$ DC du 27 juillet 1994, Loi relative au respect $d u$ corps humain et loi relative au don et à l'utilisation des éléments et produits du corps humain, à l'assistance médicale à la procréation et au diagnostic prénatal, considérant 2: "qu'il en ressort [du Préambule de 1946] que la sauvegarde de la dignité de la personne humaine contre toute forme d'asservissement et de dégradation est un principe à valeur constitutionnelle ».

11. Jean-Claude Ameisen, Pierre Le Coz (rapporteurs), Avis $n^{o} 111$ du 7 janvier 2010 sur les problèmes éthiques posés par l'utilisation des cadavres à des fins de conservation ou d'exposition muséale.

12. Dénommé ci-après «le Comité d'éthique » ou «le Comité ». 
corps plastinés ${ }^{13}$ dans une mise en scène reproduisant des activités humaines. Cette exposition décriée a été annulée suite à un référé ${ }^{14}$ intenté par l'association Solidarité Chine qui se plaignait de l'absence de traçabilité concernant l'origine de ces cadavres ainsi que du défaut ante mortem de leur consentement. Il s'agissait pour le tribunal de décrypter un discours autour d'une exposition poursuivant des fins autres que celles mises en avant, en se penchant sur le côté lucratif et spectaculaire d'une telle exhibition. Cet avis s'inscrit également dans le débat autour de la restitution de restes humains à leurs communautés d'origine, amorcé en France en 2002 avec Saartje Bartman dite la "Vénus hottentote» en Afrique du Sud et de l'indien charrua Vaimaca Perú en Uruguay ou encore par les têtes maories restituées à la Nouvelle-Zélande, lors d'une cérémonie organisée au musée du Quai Branly, le 23 janvier 2012.

Le Comité reprend certaines dispositions du rapport Léonetti qui ne limite pas la bioéthique à l'éthique des vivants mais «implique aussi de définir ce que les vivants doivent s'interdire de faire avec les corps des morts ${ }^{15}$ ». Il s'agit tout simplement d'une conception utilitariste de la mort qui se base sur les potentielles considérations du public quant aux différents usages possibles d'un corps. En effet, si d'un côté le corps vit une époque qui le libère de ses emprises sociales, de l'autre, une humanité prend conscience de ses limites au travers de la mort et souhaite borner la volonté individuelle par un standard collectif de représentation. Or, ce standard ne correspond pas forcément aux exigences de chacun en matière de dignité humaine et de représentation du corps qui «marque la clôture du sujet sur lui-même, son affirmation d'existence aux yeux des autres ${ }^{16}{ }$ et développe donc les interrogations sur son propre devenir. Ainsi, la sanctuarisation d'un corps définit les limites entre le champ d'action de la liberté individuelle et la puissance d'une collectivité agissant au nom de la représentation d'une certaine conception de la dignité. Pourtant, l'univers normatif ne se place pas forcément d'un côté ou de l'autre car «à mesure que s'accentue l'acuité

13. Le processus de plastination a été créé en 1977 par Gunther Von Hagens, anatomiste allemand, pour conserver de manière durable des parties de corps et en le montrant de l'intérieur.

14. TGI Paris, ord., 21 avril 2009, $\mathrm{n}^{\circ}$ 09/53100.

15. Rapport d'information $\mathrm{n}^{\circ}$ 2235, au nom de la mission sur la révision des lois de bioéthique (rapporteur: Jean Léonetti), disponible sur http://www.assemblee-nationale.fr/13/pdf/rap-info/i2235-t1.pdf (consulté le 21 décembre 2011), voir notamment, p. 423.

16. David Le Breton, Anthropologie du corps et modernité, 5 éd., Paris, PUF, «Quadrige », 2008, p. 36. 
des instruments de mise en valeur de l'évolution du vivant [...] s'accroît ainsi la visibilité de la continuité de la vie ${ }^{17}$ ». Ainsi, c'est dans cette continuité, par la mémoire, que s'inscrit la gestion par la conservation ou l'exposition voire par la restitution de restes humains. Pour cela, il ne s'agit pas de prôner une libéralisation de certaines pratiques, mais il apparait juste aujourd'hui d'aborder cette question par un regard frontal. C'est tout le sens qu'a apporté la loi funéraire de 2008 en insérant un article 16-1-1 au Code civil qui dispose que: «les restes des personnes décédées $[\ldots]$ doivent être traitées avec respect, dignité et décence». Ainsi, cette disposition prévoit un cadre de protection large au nom d'un principe de non-patrimonialisation du corps, étendu aux humains inanimés. Ce nouvel article, par l'alibi de protection, conditionne les restes humains en fonction des attentes éthiques d'une société et par ricochet, reconsidère le travail de recherche tout en limitant fortement, du fait de la casuistique Our Body, leur exposition. Or, comme le rappelle certains muséologues : «ce choix d'exposition ponctuelle ou permanente illustre le plus souvent la volonté de mettre en avant des valeurs symboliques spécifiques à chaque culture ${ }^{18}$ ", l'exposition de reliques religieuses dans des lieux de culte en sont la preuve. Notons encore les enjeux ${ }^{19}$ pédagogiques ou scientifiques que présentent des restes humains, d'abord dans des cabinets de curiosités puis dans les musées de médecine, tels que Dupuytren, spécialisé dans l'anatomie pathologique ou Fragonard, présentant les célèbres écorchés au musée de l'école vétérinaire de Maisons-Alfort. Les lieux tels que les musées anatomiques ou pathologiques permettent de réfléchir sur ce qui nous rend différent en tant qu'espèce et d'en apprendre davantage sur notre humanité, en ayant conscience de ce qui nous rassemble. Si les principes de protection ont donc pour finalité la dignité humaine, il devient logique de pouvoir élaborer de nouveaux critères de représentation de la mort pour assurer le traitement dans le respect que prescrit l'article 16-1-1 du Code civil. Ainsi, la question d'un repositionnement vers « un musée centré sur le corps humain, ouvert à tous les publics ${ }^{20} »$, n'est pas pour autant dénuée d'intérêt et leur destination peut donc être reconsidérée. D’une manière

17. Dominique Memmi, "Administration du vivant et sacralité », Vingtième Siècle. Revue d'histoire, $\mathrm{n}^{\circ} 87,2005-3$, p. 156.

18. Laure Cadot, En chair et en os: le cadavre au musée, Paris, École du Louvre, 2009, p. 34 .

19. Ibid., p. 35.

20. Patrice Josset, «Cachez ce sein que je ne saurais voir! L'avenir des collections anatomiques et fœto-pathologiques illustré par l'histoire du musée Dupuytren », Musées et collections publiques de France, $\mathrm{n}^{\circ}$ 259, 2010-2, p. 19. 
plus large, c'est le principe du respect des corps qui doit pouvoir être éclairci, car sa portée est encore somme toute relative sur des questions précises, par exemple, en matière d'autopsie ou d'expertises judiciaires. En effet, s'agissant de la restitution de restes humains, considérés comme des scellés biologiques, la jurisprudence oppose un refus à la famille sur le fondement du principe de non-patrimonialisation de l'article 16-1 du Code civil. Ces prélèvements ne sont envisagés qu'à la seule finalité « de recherches médico-légales pour les nécessités d'une enquête ou d'une information ${ }^{21} »$. Leur nature intrinsèque ne permet pas de les considérer comme des objets susceptibles de restitution in fine par le procureur de la République, au sens de l'article 41-4 du code de procédure pénale ou au cours de l'information judiciaire par le juge d'instruction, sur le fondement de l'article 99 du code de procédure pénale. Les prélèvements en vue d'une telle expertise concilient, en l'espèce, les risques sanitaires avec le risque d'un usage pour des motifs indignes, ce qui justifie la sélection de certaines parties du corps et la destruction des autres. Leur statut particulier ne leur retire pas la qualité de choses et permettent de nous souvenir de la sentence de Planiol, selon laquelle «les morts ne sont plus des personnes, ils ne sont plus rien ${ }^{22}$ ». Dans le cadre d'une information judiciaire donnant lieu à une autopsie, ils « sont le support d'une expertise technique ou scientifique ${ }^{23}$ ", certes sous une protection spécifique, mais dépendent principalement des nécessités de l'enquête et de l'instruction. En pratique, envisager des restitutions dans un tel cadre est difficile, même si une réflexion autour des protocoles en la matière pourrait être approfondie, tout comme sur la nature des biens restituables.

Quant à la terminologie, le Comité d'éthique préfère l'utilisation des termes « vestiges humains » à « restes humains » qui, selon lui, « renvoie à l'idée des débris et reliquats chirurgicaux voués à la destruction ${ }^{24}$ ».

21. Crim, 3 février 2010, $\mathrm{n}^{0}$ 09-83.468: «La restitution demandée porte non pas sur des objets matériels, mais sur des prélèvements humains, qui ne peuvent pas être traités comme des objets ordinaires; qu'en l'absence d'une règle de droit spécifique, la jurisprudence de la Cour de cassation a précisé que les prélèvements effectués dans le cadre d'une procédure judiciaire sur une personne vivante ou décédée ne sont pas des objets susceptibles de restitution.»

22. Marcel Planiol, Traité élémentaire de droit civil, $11^{\mathrm{e}}$ éd., Paris, LGDJ, 1928, p. 144.

23. Crim, 18 janvier 2011, $\mathrm{n}^{\circ}$ 10-83.386 : après des observations sur le dogme catholique, la cour considère "que la non-restitution des prélèvements effectués lors de l'autopsie n'est donc pas une atteinte à la liberté de penser, de conscience et de religion définie et protégée par l'article 9 de la Convention européenne des droits de l'homme».

24. Jean-Claude Ameisen, Pierre Le Coz, op. cit., p. 10. 
Nous retiendrons davantage les termes de "restes humains" car ils assurent, par leur aspect générique, une représentativité de toutes les différentes variables et composantes de la mort que peuvent représenter les fragments, les traces, le cadavre, le squelette, les reliques, la dépouille ou les vestiges. Ces derniers ont une connotation plus archéologique au sens large et ne désignent pas forcément quelque chose d'humain ou sinon une chose de datée dans le temps qui ne semble pas approprié pour des restes vieux seulement d'un ou deux siècles. De plus, la définition varie en fonction des pratiques ou de la vision du monde de chacun mais également de la langue. En anglais, par exemple, est couramment employée l'expression «human remain » que l'on pourrait traduire, en fonction du contexte, par reste, vestige ou dépouille. La langue française nous oblige à choisir les termes "restes humains " qui couvriront un champ plus large de connotations. En effet, si un cadavre est un reste humain dans sa composante charnelle, la réciproque n'est pas vraie et le terme relique est instantanément disqualifié de par sa connotation religieuse. Si l'appellation «restes humains " n'est pas forcément la meilleure, elle s'avère finalement la plus pertinente car elle englobe tout autant les cadavres que les vestiges, les squelettes ou encore les fragments de crânes, de têtes ou d'os.

La question du devenir muséal des restes humains est un moyen parmi d'autres d'organiser le vivre ensemble à «l'époque de la globalisation où les tensions entre village global et archipel planétaire renvoient aux débats $^{25}$ » entre l'universel et le relatif, où seule une approche interculturelle demeure un horizon souhaitable. Il s'agit de faire vivre les diverses interrogations sur ce sujet par une réelle dialogie, c'est-à-dire par un dialogue transcendant les logiques en présence, d'acteurs aux aspirations différentes. À ce titre, il est utile de s'interroger plus globalement sur la manière la plus efficace d'appréhender les problématiques sur la mort et le corps exposé. Les objectifs scientifiques, les considérations juridiques doivent pouvoir être remises en perspective, notamment sur la question du devoir des personnes et des peuples envers leurs morts. En substance, il s'agit de s'interroger sur l'accroissement des mesures de protection autour du corps, mort ou vif, et dans quelle mesure la dignité humaine peut être le fondement du principe de non-patrimonialisation. La critique de l'avis du Comité d'éthique se veut, dans un premier temps, comme un instrument de réflexion, à partir de pratiques muséales

25. Christoph Eberhard, «Trois problématiques pour une dynamique d'anthropologie du droit ", Cahiers d'anthropologie du droit, Juridicités, hors-série, Laboratoire d'anthropologie juridique de Paris (LAJP), Paris, Katharla, 2006, p. 65. 
et de propositions juridiques qui ne réfutent pas systématiquement la présence du cadavre au musée, tant au niveau de la représentation ou de la conservation, mais qui constituent des degrés différents de préoccupations. La seconde partie de l'analyse se centrera sur la question de la restitution des restes humains en lien avec leur statut juridique et son adaptabilité avec le principe de dignité humaine, notamment dans le cadre de leur présence au sein de musées mettant en œuvre une nouvelle variable, la notion de domaine public. À ce titre, une étude comparative permettra de mettre en perspective une diversité de solutions, qui ont pour but un dépassement de ces questions passionnelles autour de la simple dichotomie entre conservation ou restitution.

\section{Le cadavre au musée: quand conserver n'est pas jouer}

Le Comité distingue deux types d'usage posthume des êtres qui sont destinés à la conservation ou l'exposition : un usage actuel des cadavres dans les salles d'exposition et la question du devenir des "vestiges» humains dans les collections face aux diverses revendications culturelles. Dans un premier temps, il convient, notamment par l'étude de l'affaire Our Body, d'appréhender les contextes possibles d'une exposition de restes humains (1.1), que l'on pourra confronter à l'argumentaire scientifique, permettant d'expliciter ce que peut recouvrir, pour leur conservation, la notion de l'article 16-1-1 du Code civil de traitement dans la dignité (1.2).

\subsection{Détour vers une rencontre de restes humains lors d'expositions publiques}

Cette rencontre du public face à des restes humains ne doit pas se faire dans n'importe quelle condition puisqu'une éthique de l'exposition du corps est actuellement au centre d'un débat (1.1.1) dans lequel la construction de critères, à la suite de l'affaire Our Body, est devenue indispensable pour mener à bien une telle entreprise (1.1.2).

\subsubsection{Une éthique de l'exposition au centre d'un débat}

Le rapport du Comité constate que les expositions de cadavres «se déroulent dans différents États occidentaux de tradition libérale ${ }^{26}$ ", mais cette pratique est depuis longtemps institutionnalisée au sein de notre

26. Jean-Claude Ameisen, Pierre Le Coz, op. cit., p. 4. 
culture avec comme nous l'avons vu, le culte voué aux différentes reliques, présentées aux fidèles en développant un aspect de mise en scène, lorsque celles-ci sont visibles à tous. L'argument de l'interdit culturel de l'exposition de cadavres ne peut, en lui-même, être déconnecté d'un contexte éthique qui naît d'un décalage entre les conduites sociales et la règlementation juridique dans lequel s'insère ce qui échappe "aux systèmes et aux codes et les transcendant tout en y [...] orient[ant] néanmoins [...] la conduite humaine ${ }^{27}$ ». C'est pourquoi il revient à notre société de faire la part des choses entre la profanation et l'exhumation qu'elle "vit comme une transgression choquante ${ }^{28}$ " et la conservation ou l'exposition de restes humains dans un cadre muséal et scientifique. Il ne s'agit précisément pas des mêmes niveaux d'approches, car si la profanation de tombes et de cadavres est constitutive d'un délit pénal ${ }^{29}$, l'exhumation ne peut être ordonnée que par une décision de justice ${ }^{30}$ ou par des dispositions relatives aux concessions funéraires et gérées au niveau communal ${ }^{31}$. Ainsi, hormis la possibilité de donner son corps à "un établissement de santé, de formation ou de recherche ${ }^{32}$ », les dépouilles sont soustraites du regard public et ne peuvent être qu'uniquement enterrées ou incinérées ${ }^{33}$. La seule possibilité s'agissant de l'utilisation de tout ou partie d'un corps réside dans un cadre d'un prélèvement d'éléments du corps humain en vue d'un don et se limite à un l'usage médical ou scientifique, tel que prévu par la loi ${ }^{34}$. Une fois cette distinction établie entre exhumation et conservation, il apparaît donc comme tout à fait raisonnable, dans le cadre légal ainsi posé, qu'une exposition puisse faire l'objet en amont d'une information publique par les établissements souhaitant effectuer ce type de démarche. Cette dernière se réalise alors que le cadavre n'a pas fait l'objet une inhumation initiale et seulement autour d'un projet scientifique établi

27. Guy Bourgeault, "L'éthique et le droit face à la technologie», Revue Juridique Thémis, $\mathrm{n}^{\circ} 22 / 3,1988$, p. 282.

28. Jean-Claude Ameisen, Pierre Le Coz, ibid.

29. En vertu des articles 225-17 à 225-18-1 établis à la section IV du chapitre v du titre II du livre deuxième du Code pénal: «Des atteintes au respect dû aux morts. »

30. CA Paris, 6 novembre 1997, n XPo61197X, Recueil Dalloz, 1998, p. 122, observations Philippe Malaurie.

31. Article L 2223-17 du Code général des collectivités territoriales, s'agissant des concessions non renouvelées.

32. Article R2213-13 du CGCT.

33. Article R2213-15 du CGCT: "Avant son inhumation ou sa crémation, le corps d'une personne décédée est mis en bière », la cryogénisation est, de plus, illicite: CE, 6 janvier 2006, M. Rémy M. et autres, $\mathrm{n}^{0} 260307$.

34. Articles L 1211-1 à L 1211-9 du Code de la santé publique. 
ou d'institutions spécialisées. Si «nous ne savons rien de l'histoire de ces personnes réduites à leur corps ${ }^{35}$ ", c'est tout l'enjeu d'une exposition qui souhaite présenter des garanties d'éthique que d'en informer le public. En effet, il serait juste de revenir sur l'histoire de ces corps exposés, en ne présentant pas simplement une tête ou un décharné mais en permettant, par un encart ou une liste en début d'exposition, de raconter l'histoire des personnes présentement exposées. Il serait également utile de réfléchir sur la possibilité de nommer les corps non plus comme des êtres désincarnés mais par leur identité ante mortem en faisant prendre conscience au visiteur de la portée de ce qu'il regarde. On peut enfin penser aux possibilités de moulage dans les cas qui ne permettent pas l'effectivité de l'exposition et la durabilité de la conservation. Ainsi, au lieu d'un cadavre "passe-partout», le cadavre serait présenté comme un témoin de l'histoire d'un patrimoine commun de l'humanité. En souhaitant éviter à d'autres l'exposition de leurs restes sans leur consentement, la Cour de cassation a voulu utiliser l'article 16-1-1 du Code civil pour imposer des limites en considérant "que l'exposition de cadavres à des fins commerciales méconnaît cette exigence ${ }^{36} »$ de traitement dans le respect. Cela signifie a contrario qu'une exposition à des fins non commerciales pourrait être possible dès lors que le consentement de la personne aurait été préalablement reçu. En effet, l'argumentaire sur le consentement trouve ses limites dans le discours scénographique en ayant conscience qu' "une civilisation qui commence doit d'abord poser ses règles de conduite vis-à-vis des morts ${ }^{37}$ ». Si la liberté consiste à pouvoir faire tout ce qui ne nuit pas à autrui, en vertu de l'article 4 de la Déclaration des droits de l'homme, il apparait, en l'espèce, qu'une personne ne pourra être autorisée à ce que son corps soit exposé, uniquement si elle remplit les conditions de l'article 16-1-1 du Code civil, hors d'un cadre commercial, en vertu du principe de non-patrimonialisation de la personne humaine, vivante ou morte. Il s'agit donc de déterminer, avant d'exposer quiconque, l'ensemble des potentialités sur un reste humain: ce qui pouvait être admis auparavant trouve aujourd'hui ses limites mais cela ne doit pas, pour autant, devenir un problème insurmontable.

35. Jean-Claude Ameisen, Pierre Le Coz, op. cit., p. 5.

36. $\mathrm{Civ}_{1}{ }^{\mathrm{re}}, 16$ septembre $2010, \mathrm{n}^{\mathrm{o}}$ 09-67.456.

37. Philippe Muray, Le XIX ${ }^{e}$ siècle à travers les âges, Paris, Gallimard, «Tel», 1999, p. 45. 


\subsubsection{La nécessité de construire des critères rigoureux}

L'affaire Our Body a cristallisé les problèmes que pouvaient susciter de telles expositions en mettant en lumière une distinction ${ }^{38}$ entre celles qui ont au sein d'un musée public un souci de vérité anatomique ou pathologique, dans un but d'information pédagogique et celles qui sont à l'initiative d'institutions privées. Ces dernières sont considérées comme pouvant favoriser un certain axe éditorial visant au spectaculaire de la manifestation. Dans ce genre d'exposition, où les cadavres ont subi un processus de plastination, il est exact que le consentement ne doit pas être la seule donnée la permettant effectivement, mais les organisateurs doivent tout mettre en ouvre pour éviter que le cadavre soit mis dans une position de spectacle sans contextualisation historique. À l'instar des objets-ambassadeurs, les restes humains exposés peuvent être le témoignage d'une histoire, d'un héritage culturel que l'on présente dans un autre temps et lieu pour une autre histoire dans le cadre d'une exposition. Il en serait de même pour un cadavre « du cru » car même si son origine culturelle se rapproche de ses contemplateurs, en supposant qu'aucun visiteur étranger ne sera présent, son histoire pourra faire l'objet d'une mise en situation, en fonction de la thématique d'exposition. La mort est bien la continuité de la vie et nous avons déjà constaté que le droit "n'abandonne pas l'homme après sa mort ${ }^{39}$ " mais le fait changer de statut, de personne en chose. La dimension ancestrale ou le retour à la poussière sont des notions construites et leur représentation au moyen d'une exposition permet de construire les éléments constitutifs de notre humanité. L'aspect spectacle de la mort que présentait l'exposition Our Body et une démarche conduisant à réfléchir autour d'une présentation respectueuse ne sont ni du même ordre ni du même niveau dans la vision et les attentes possibles du spectateur. Dans le dernier cas, il s'agit de faire apparaître une médiation entre un regard vers la mort par les vivants et une scénographie visant au principe du respect des morts, comme le suggère l'article 16-1-1 du Code civil. On ne peut assimiler une exposition qui serait contraire aux volontés ou au respect nécessaire sur un reste humain, tout particulièrement lorsque celui-ci fait partie des stigmates de l'histoire, notamment coloniale ${ }^{40}$, à une exposition qui aurait pour ambition de nous apporter un témoignage

38. Opéré particulièrement dans CA Paris, 30 avril 2009, n 09/09315.

39. Jean-Pierre Gridel, "L'individu juridiquement mort », Recueil Dalloz, 2000, p. 266.

40. Le squelette de la Vénus hottentote a été retiré de la vue du public en $1976 \mathrm{au}$ musée de l'Homme. Voir, pour son historique : Gérard Badou, L'Énigme de la Vénus hottentote, Paris, Payot et Rivages, 2002, 188 p. 
sur notre propre humanité. La limite peut s'avérer aujourd'hui, dans notre contexte juridique et législatif, ténue mais elle n'est pas pour autant indépassable puisqu'elle se base sur la finalité première de la valeur marchande ou esthétique d'une telle exposition. Notre jurisprudence sur l'affaire Our Body a été claire sur le principe de non-patrimonialisation mais hésitante sur ses contours. En effet, le juge a bien rappelé en première instance que la destination des restes humains demeure la crémation ou l'inhumation tout en précisant que «la détention privée de cadavres est illicite $^{41}$ ». Cependant, la société Encore Events, organisatrice de l'exposition a interjeté appel de la décision, ce qui a permis à la Cour ${ }^{42}$, tout en retenant la violation manifeste de l'article 16-1-1, d'orienter son paradigme autour du consentement en considérant que si celuici est recueilli et si l'exposition contient une finalité «scientifique ou pédagogique ${ }^{43}$ ", elle pourra s'organiser au nom de la liberté d'expression et du droit au savoir. Cette brèche fut aussitôt refermée par la Cour de cassation dans son arrêt du 16 septembre 2010 qui prononça sèchement l'interdiction de l'exposition de cadavres à des fins commerciales sur le fondement du même article. Il existe une évolution jurisprudentielle et la question principale reste de savoir si, aujourd'hui, une exposition de cadavres dont l'accessoire serait commercial, comme les ventes d'entrées dans un musée ou l'achat d'un catalogue d'exposition, mais dont le principal serait le contenu, donc la finalité scientifique ou pédagogique, rentrerait en conformité avec l'article 16-1-1 du Code civil et l'interprétation qu'en a déterminé la Cour de cassation. L'étude de ce cas est particulièrement révélateur du fait qu'aujourd'hui les cadavres «sont nimbés d'une aura, d'un prestige, d'un respect qui nous plongent dans la plus grande perplexité, tout se passe comme si [...] le corps mort reprenait la grandeur, la dignité que le corps vivant avait perdu ${ }^{44}$ ". Ainsi, les institutions muséales se doivent d'être à l'écoute d'un public qui prend conscience de ce qu'un corps mort peut devenir et à quel niveau il peut se placer dans la nature. Il ne s'agit pas de prôner un naturalisme contre l'humanisme, mais d'accorder davantage d'attention

41. TGI Paris, ord., 21 avril 2009, $\mathrm{n}^{\circ}$ 09/53100.

42. CA Paris, 30 avril 2009, $n^{0}$ 09/09315: «La protection du cadavre et le respect dû à celui-ci commandent tout d'abord de rechercher si les corps ainsi exposés ont une origine licite et s'il existe un consentement donné par les personnes de leur vivant sur l'utilisation de leur cadavre.»

43. Ibid.

44. Bernard Edelman, Ni chose ni personne, Paris, Herman, 2009, p. 70. 
aux potentialités d'un contrat naturel ${ }^{45}$. À cet égard un mélange entre l'artificiel au moyen de moulages et la réalité d'un corps exposé peut également être une réponse pour permettre au public de se placer dans une dimension comparative, tout en lui soumettant une distance critique nécessaire dans le cadre de la subjectivité des émotions autour de la représentation de la mort. Dans notre espace juridique et éthique actuel, il devient donc difficile d'organiser ce genre de manifestation. Le souci premier des organisateurs, une fois les conditions de traçabilité du cadavre réunies, est d'exprimer clairement le but de la manifestation en évitant le mélange des genres ${ }^{46}$ entre les dimensions dites pédagogiques ou scientifiques et d'autres présentant un cadre privilégiant des aspects spectaculaires ou artistiques.

\subsection{L'affirmation du contenu du traitement dans la dignité par la conservation scientifique des restes humains}

Les arguments quant à la conservation des restes humains dits archéologiques ou plus récents ne sont pas dénués d'intérêt (1.2.1) et permettent de développer les potentialités de ce que recouvre le principe de traitement dans la dignité, tel qu'affirmé à l'article 16-1-1 du Code civil (1.2.2).

\subsubsection{Les arguments pour une conservation scientifique}

Cette tradition d'étude, amorcé, dans son caractère scientifique à la Renaissance par Vésale ${ }^{47}$, s'emploie au moyen de pratiques anatomiques à cartographier le corps humain et à transmettre concrètement son fonctionnement auprès des générations d'étudiants de médecine. Le Comité n'oublie donc pas l'aspect de recherche sur des restes humains, tout en le limitant fortement, en expliquant que «les scientifiques ont besoin de ces corps [préhistoriques] pour les étudier et comprendre l'évolution des espèces ${ }^{48}$ ". Il ne conçoit donc qu'un cadre d'étude pour des restes humains plus anciens et ne porte pas son regard sur les recherches faites par des équipes de chercheurs avec des techniques modernisées. L'argument du savoir anatomique sur le corps physique

45. Michel Serres, Le Contrat naturel, Paris, Flammarion, "Champs, essais », 1992, $191 \mathrm{p}$.

46. Comme le rappelle l'avis défavorable du Comité d'éthique sur l'exposition Our Body, du 10 juin 2008 : «Une atteinte à la dignité humaine».

47. André Vésale, De Humani corporis fabrica libri septem, Basileae, 1555, 882 p.

48. Jean-Claude Ameisen, Pierre Le Coz, op. cit., p. 12. 
même est contourné par le Comité par l'utilisation de simulateurs médicaux reproduisant virtuellement les caractéristiques humaines. Une des limites de ces techniques peut provenir du fait que l'on ne voit plus que par la technique de modélisation virtuelle empiétant ainsi sur le réel d'une chair, d'un cœur, d'un organe. En considérant cet aspect des choses, il apparait légitime de se demander dans quelle mesure le Comité d'éthique fournit une réponse adéquate sur la sanctuarisation des corps, conduisant les facultés de médecine à limiter l'approche anatomique traditionnelle. Cela pourrait se traduire à terme par une lignée de médecins dont l'apprentissage serait dénué de contact avec un corps tel qu'exposé lors de leçons d'anatomie. Toutefois, il revient au professionnel de recenser en la matière quels peuvent être les besoins actuels et s'ils peuvent ou non être remplacés d'une manière ou d'une autre. Pourtant, de manière plus large que les études médicales, de nombreux chercheurs et disciplines travaillent quotidiennement sur des restes humains, pour lesquels le contact avec le réel est nécessaire. Par exemple, au sein du musée de l'Homme, les chercheurs invités sont libres de faire des études sur des cas spécifiques en ayant les moyens d'une visée comparative du fait de l'importance de la diversité biologique, avec près de 160 pays représentés pour un total recensé de 17679 crânes, 975 squelettes et 80 momies. Un bon nombre d'informations se trouvent dans l'os lui-même et l'étude de l'ADN ancien, dont les premiers travaux ne remontent qu'à une vingtaine d'années, permet de raconter la vie de ces personnes, de retracer leur histoire, leur parenté et les mouvements de population qui leur sont associés à travers ces analyses. Un des autres multiples enjeux de la conservation des restes humains, recouvre l'ensemble des problématiques liées à la paléopathologie. En effet, cette discipline aux multiples branches étudie les traces de maladies contenues dans les restes humains pour permettre de découvrir leurs origines, leurs développements historique, géographique, alimentaire, sanitaire et retrace des hérédités entre squelettes. Ayant eu son âge d'or au début du $\mathrm{xx}^{\mathrm{e}}$ siècle, notamment grâce à des radiographies de momies, la paléopathologie et la génétique d'aujourd'hui sont des disciplines qui permettent d'apporter une "dimension médico-historique générale en montrant que la connaissance historique des maladies contribue à une meilleure appréciation de la pathologie d'aujourd'hui ${ }^{49}$ ». Il s'agit de conduire une réflexion entre les restes humains anciens et contemporains pour

49. Pierre Charon, «Histoire de la paléopathologie», dans Philippe Charlier (dir.), Ostéo-archéologie et techniques médico-légales, tendances et perspectives. Pour un manuel pratique de paléopathologie humaine, Paris, De Boccard, 2008, p. 22. 
permettre, par des lignes directrices établies entre musées, de concevoir une charte éthique affirmant les objectifs de conservation et de travail en la matière. Notons que les collections muséales disposant d'une série de restes humains, sont généralement non identifiables et à l'origine incertaine, posant moins de difficultés quant à leur conservation. En effet, ce n'est pas la même chose d'avoir sous sa garde des restes humains d'un peuple toujours vivant et d'un peuple qui n'existe plus ou dont le temps a effacé toute trace d'une localisation fiable. Il s'avère donc nécessaire de «combiner écoute attentive et souci de conservation [et] [...] mettre en balance les impératifs scientifiques et les revendications des communautés indigènes ${ }^{50}$ ». Un travail d'identification doit donc pourvoir être réalisé dans un souci de diffusion de la connaissance par des chercheurs qui ont à l'esprit qu'ils ne peuvent travailler de la même manière sur des restes humains dits archéologiques et ceux dont la mémoire est constitutive d'un élément de l'histoire coloniale, perçu aujourd'hui comme un élément persistant de domination d'une culture sur une autre. C'est tout le sens de la disposition prévoyant un traitement dans la dignité pour les restes humains, pour apporter un gage de protection efficace sans pour autant les associer matériellement au principe de dignité humaine, qui est, comme nous le verrons, réservé aux êtres humains vivants.

\subsubsection{L'affirmation du principe d'un traitement dans la dignité}

L'article 16-1-1 a souhaité préciser les conditions d'encadrement de protection du corps humain en l'étendant aux restes et aux cendres qui «doivent être traitées avec respect, dignité et décence». En somme, la primauté de la personne dans ce qui la constitue fondamentalement, déjà affirmée à l'article 16 du Code civil qui «interdit toute atteinte à la dignité de celle-ci et garantit le respect de l'être humain dès le commencement de sa vie », trouve son prolongement pour les restes humains, considérés comme méritant également une protection particulière. Ce principe a $\mathrm{pu}$ trouver sa résonnance dans la jurisprudence qui avait estimé que la publication dans la presse de photos ${ }^{51}$ d'un homme assassiné était contraire à sa propre dignité et poursuit, dans le même registre, en

50. Alain Froment, «Ne liquidons pas les collections anthropologiques", La Recherche, $\mathrm{n}^{\mathrm{o}} 420$, juin 2008, p. 96.

51. Civ. $1^{\text {re }}, 20$ décembre 2000, $\mathrm{n}^{0} 98-13.875$ : «la photographie publiée représentait distinctement le corps et le visage du préfet [Erignac] assassiné [...] cette image était attentatoire à la dignité de la personne humaine». 
devenant le résultat ${ }^{52}$ de l'atteinte à la mémoire du mort et donc à la vie privée de la famille. Il convient toutefois de constater qu'il ne s'agit pas exactement de la même situation puisque dans la première espèce, la photographie représentait un corps déjà mort, alors que dans la seconde, ce n'était qu'une menace de ses ravisseurs, malheureusement concrétisée. Toutefois, notons qu'entre ces deux arrêts, pour expliquer cette continuité, l'article 16-1-1 est venu consacrer un droit au traitement des restes humains dans la dignité et non une dignité en soi de l'homme après la mort, comme le suggérait la première espèce. Nous pouvons nous questionner alors sur le sens de la dignité humaine au vu de l'utilisation rapprochée qu'en fait la Cour de cassation dans ces deux arrêts, qu'il s'agisse de la représentation d'un homme pris sous la menace, dans un état de torture et d'un homme déjà mort. La dignité humaine s'appliquerait dans les deux espèces sur le fondement de la vie privée et familiale. La Cour de Strasbourg s'est contentée, quant à elle, toujours dans l'affaire concernant la photographie du préfet Érignac, d'apprécier le respect des émotions de la famille et de leur vie privée, mais ne reçoit pas l'atteinte à la dignité pour des images publiées d'un corps sans vie ${ }^{53}$. Ainsi, si le principe de dignité peut être exploité, s'agissant de l'être humain après la mort pour sa représentation photographique, puis sa publication, par le biais de l'atteinte à la vie privée et familiale, la réflexion évolue désormais autour de cet aspect de traitement dans le respect. Cette notion n'est plus constitutive d'une qualité commune, prérequis à tous les humains que constitue la dignité humaine, mais doit être utilisée comme grille d'analyse pour ceux qui utilisent des restes humains à des fins scientifiques ou culturelles. Il apparaît donc que le principe de l'article 16-1-1 n'existe que pour faire prendre conscience aux divers professionnels que l'étude ou la

52. Civ. $1^{\text {re }}, 1^{\text {er }}$ juillet 2010, $\mathrm{n}^{\mathrm{o}}$ 09-15.479: la famille de Ilan Halimi a assigné la société éditrice du magazine incriminé pour avoir publié une photographie le représentant bâillonné et entravé, la cour a considéré cela comme « contraire à la dignité humaine, [et] constituait une atteinte à la mémoire ou au respect dû au mort et dès lors à la vie privée des proches".

53. CEDH, 14 juin 2007, Hachette Filipacchi associés c/. France, $\mathrm{n}^{\circ} 71111 / 01$. La Cour estime qu'il n'y a pas eu de violation de la liberté d'expression du magazine, tel qu'entendu au sens de l'article 10 de la Convention, et considère que l'injonction de publier un communiqué attestant de la violation de la vie privée des proches de la victime constitue « dans son principe comme dans son contenu, [...] la sanction emportant le moins de restrictions à l'exercice des droits de la société requérante sur l'échelle des sanctions rendues possibles par la législation interne, notamment au regard de l'interprétation des dispositions de l'article 9 alinéa 2 du Code civil par les juridictions françaises » $(\$ 61)$. 
présentation de restes humains doit être pensée dans un cadre digne et de nombreux exemples mettant en œuvre ces prescriptions existent. Ainsi, le musée de l'Homme se préoccupe tout particulièrement de la conservation de ses collections: les restes humains sont entreposés dans un local à moins de $40 \%$ d'humidité, avec une température toujours inférieure à 18 degrés. S'agissant des pratiques d'exposition, le château-musée de Boulogne-sur-Mer détient dans ses collections une momie qui est recouverte d'un drap chaque soir à la fermeture, et qui n'est pas visible lorsque les très jeunes enfants viennent en visite scolaire au musée. Au musée Bargoin de Clermont-Ferrand, la même problématique s'est posée sur la manière de montrer leur momie, à l'occasion d'une exposition temporaire achevée en mai 2010. L'exposition de la momie a suscité un engouement local et l'affaire Our Body ayant eu un certain écho, le musée Bargoin a instauré « deux types d'accès, un accès payant pour les expositions ne présentant pas de restes humains et un accès libre pour les restes humains ${ }^{54}$ ", situé dans un espace actualité. Cela restait envisageable car ce musée peut se financer par ses expositions permanentes mais ce serait difficilement concevable au musée Dupuytren, dont la conception est bien la présentation pédagogique des dimorphismes anatomiques. Ces exemples illustrent bien que le corps au sein des musées ne peut s'apparenter à un bien culturel $^{55}$ comme un autre et que le montage de toute exposition intégrant des restes humains doit passer par des réflexions autour du discours employé, pour savoir si toutes les présentations peuvent, dans notre contexte actuel, être admissibles. Il apparaît nécessaire de réfléchir sur les conditions d'ouverture de telles expositions en posant des règles séparant, par exemple, les restes humains d'autres biens culturels, en posant une limite d'âge ou en avertissant du contenu et de l'objectif de l'exposition dès l'entrée. Cette prise de conscience sur la préservation, l'exposition, la recherche ou la restitution de restes humains, tant de la part des professionnels que du Comité d'éthique, permet une réflexion accrue sur ce sujet pour ceux qui en ont la charge quotidienne. Cette évolution ne doit pas pour autant être génératrice d'une vague moralisatrice, qui justement au nom de la dignité humaine refuse, par principe, toute recherche ou toute exposition de restes

54. Hélène Déchamps-Rougeron, Exposition publique et restes humains. Enjeux juridiques et questions pratiques, rapport de stage du $1^{\mathrm{er}}$ mars 2010 au 31 mai 2010, Clermont-Ferrand, M2 droit du patrimoine historique et culturel, p. 22.

55. Au sens du régime de protection du titre I et d'acquisition du titre II du livre $\mathrm{I}^{\mathrm{er}} \mathrm{du}$ code du patrimoine. 
humains. D'un côté, il semble aujourd'hui possible d'exprimer que le principe de dignité humaine permet de protéger ${ }^{56}$ la personne dans des situations qui la placent dans des conditions d'indignité de son vivant ou d'assurer le respect dû aux morts, sous le fondement de la protection de la vie privée et familiale. De l'autre, la dignité humaine ne pouvant être exprimée s'agissant des restes humains uniquement par sa famille au nom de l'atteinte à la vie privée, il conviendrait de changer de paradigme en permettant l'effectivité d'une protection dans le respect, au nom d'une dignité de l'humanité. Cela comprendrait tant le respect des restes humains, des animaux que la prévention et la réparation aux dommages de l'écosystème, c'est-à-dire tous ceux qui ne disposent pas d'une protection efficace au moyen d'une personnalité juridique et qui seraient protégés par une instance tierce ${ }^{57}$, leur servant de garantie de représentation. En effet, consacrer une dignité de l'humanité permettrait d'intégrer le vivant et la mémoire en considérant que sa préservation ne se limite pas à celle de l'Homme et en déterminant, à l'inverse, que la dignité humaine «commande d'appréhender l'homme, à la fois dans son individualité et ses droits de personnalité, et dans son humanité consubstantielle ${ }^{58} »$. Disposer de deux conceptions de protection autour du principe de dignité permettait de consacrer le fait que «la vie ne cesse de rencontrer des messies parmi les choses et les êtres, comme brin de paille parmi des milliards d'aiguilles d'or [...] en Biogée, il suffit de se baisser pour les ramasser $[\ldots]$ nous vivons comme des anges en attente de merveilles ${ }^{59}$ ", nous commencerons donc une nouvelle étape dans notre condition humaine.

La question de la conservation des restes humains a donc permis d'importants développements autour de ce que peut recouvrir l'article 16-1-1 et comment celui-ci peut compléter le principe de dignité humaine. Il convient désormais de revenir sur le sens général de ce principe et

56. Le chapitre v du titre II du livre deuxième du code pénal « Des atteintes à la dignité de la personne ", comprend, outre les atteintes dû au respect des morts, la répression des discriminations, la traite des êtres humains, le proxénétisme, le recours à la prostitution de mineurs, l'exploitation de la mendicité, les conditions de travail et d’hébergement indignes, ainsi que le bizutage.

57. Évoquée depuis le sommet de Rio de 1992, une Organisation mondiale de l'environnement, aux compétences élargies, pourrait jouer ce rôle ou encore une sorte d'exécuteur testamentaire dans des conditions similaires à celles posées aux articles 1025 à 1034 du Code civil.

58. Sylvie Wallaert, «Entre permanence et transformation: la force et l'avenir de la dignité humaine", Revue de la recherche juridique, 2010-2, p. 676.

59. Michel Serres, Biogée, Paris, Éditions-dialogues.fr, 2010, p. 160. 
d'analyser dans quelle mesure celui-ci peut servir comme un élément de réponse à la restitution des restes humains à leurs peuples d'origine.

\section{L'expression du principe de dignité humaine soluble dans les politiques de restitution}

L'enjeu principal se concentre dans le fait de savoir si, une fois le principe de dignité humaine explicité comme mettant en valeur la protection de l'être humain vivant, il peut être employé pour justifier la restitution de restes humains faisant partie du domaine public (2.1). L'analyse du droit comparé en la matière, et notamment anglais du fait de notre proximité coloniale historique, permettra ensuite d'envisager sous un autre angle cette question des restitutions et de chercher enfin à savoir comment cette problématique pourrait être dépassée (2.2).

\subsection{Retour sur le sens de dignité « humaine» et son adaptation au domaine public}

Ce principe aux multiples facettes, la dignitas, les caractéristiques que doivent présenter un individu pour être digne face à un autre est désormais admis dans notre droit, même s'il convient de lui donner tout son sens en ne le dévoyant pas (2.1.1). Cela permettra ensuite de mettre en évidence les difficultés d'une politique de restitution cohérente en France, liées, entre autres, au nom du principe d'inaliénabilité des collections publiques (2.1.2).

\subsubsection{La construction d'un principe fondamental}

L'essor du principe de dignité pour la personne humaine, envisagée dans son sens holiste, peut se concevoir comme l'imposition par l'État d'une conception morale de la société vis-à-vis des comportements individuels au sein des espaces privés. Ces derniers participent à cette « incontournable internormativité ${ }^{60}$ » dans laquelle les codes sociaux, la moralité ou encore l'éthique sont envisagés par la sphère étatique. L'État, en suivant l'évolution des comportements sociaux, a tendance à légiférer sans savoir où poser la limite et impose in fine un interdit général, comme nous avons pu le constater avec l'exposition des corps. La dignité, par sa dimension intersubjective, permet la création d'un cadre social commun mais dont personne ne peut identifier formellement, plus

60. Jean-Guy Belley, "Le pluralisme juridique comme orthodoxie de la science du droit », Revue canadienne Droit et société, vol. 26, nº 2, 2011, p. 264. 
que pour n'importe quel autre concept, ses contours précis. L'essor récent de cette notion juridique s'accorde toutefois à énoncer au moins que la dignité humaine s'apparente avant tout à celle des vivants, même si certains envisagent de l'étendre, comme nous le verrons, aux dépouilles mortelles. Pour l'instant, le droit considère que ce principe existe pour permettre aux individus de vivre dans des conditions jugées comme un standard minimum de dignité pour tous. Ainsi, la loi et la jurisprudence ont $\mathrm{pu}$, à certaines occasions, justifier une interdiction de travail ${ }^{61}$, affirmer la nécessité d'un logement pour des personnes vulnérables ${ }^{62}$, le traitement sous conditions d'une personne malade ${ }^{63}$, le harcèlement moral ${ }^{64}$ ou sexuel ${ }^{65}$ dégradant les conditions de travail ou encore l'accomplissement d'une peine de prison ${ }^{66}$ par ce concept de dignité humaine, prérequis d'attentes sociales protectrices communes. Ce principe est inhérent à la personne même du fait que son humanité est reconnue comme telle au sens propre et dégagée de toutes considérations transhumanistes ${ }^{67}$. L'enjeu réside donc dans le fait de savoir si le cadre de protection qu'offre le principe de dignité humaine peut persister pour les restes humains et donc, du fait de sa nature constitutionnelle, venir parasiter le principe d'inaliénabilité des collections publiques. Il semble pourtant difficile de faire coïncider un même concept dans ces deux étapes de l'existence humaine qui offrent des solutions juridiques différentes. En effet, la raison principale de cette dissociation vient du fait

61. CE, ass., 27 octobre 1995, $\mathrm{n}^{\circ} 143578$ et $\mathrm{n}^{\circ}$ 136727, Ville d'Aix-en-Provence; Commune de Morsang-sur-Orge.

62. CE, réf., 10 février 2012, $\mathrm{n}^{\circ}$ 356456, Karamoko F. c/. ministre des Solidarités et de la Cohésion sociale: reconnaissance d'un droit à l'hébergement d'urgence comme liberté fondamentale et dont la dignité humaine est la composante.

63. Article L 1110-2 Code de la santé publique: «la personne malade à droit au respect de sa dignité »; et L 1111-4 al. 2 : «le médecin sauvegarde la dignité du mourant et assure la qualité de sa fin de vie »; et voir notamment CE, ass., 2 juillet 1993, Milhaud, $\mathrm{n}^{\mathrm{o}} 124960$.

64. Soc., 24 février 2008, $M^{m e}$ Gerin, $\mathrm{n}^{0}$ 06-45.579: « ... peuvent constituer un harcèlement moral des agissements ayant pour objet ou pour effet une dégradation des conditions de travail susceptibles de porter atteinte aux droits et à la dignité du salarié...»

65. Article 222-33 du Code pénal, issu de la loi du 06 août 2012 relative au harcèlement sexuel.

66. Pacte international relatif aux droits civils et politiques du 15 décembre 1966, art. 10 al. 1: "Toute personne privée de sa liberté est traitée avec humanité et avec le respect de la dignité... » et son principe est reconnu pour borner les injonctions du personnel pénitentiaire, voir notamment CE, 20 mai 2011, $\mathrm{n}^{0} 326084$.

67. Marie-Angèle Hermitte, «Analyse juridique du transhumanisme», dans Guillaume Canselier, Sonia Desmoulin-Canselier (dir.), Les Catégories ethno-raciales à l'ère des biotechnologies, Paris, UMR de droit comparé de Paris, 2011, vol. 24. 
de la perte de la personnalité juridique de l'être humain après la mort. Le sujet de droit qu'il était ne peut, en son nom, ester en justice pour réparer les atteintes qui pourraient être commises sur lui-même mais doit compter sur sa famille qui peut poursuivre, comme nous l'avons mentionné, au nom de l'atteinte à la vie privée. Si vivant, on peut objecter sur la recevabilité de la dignité humaine dans un cas d'espèce; mort, on est condamné à une application forcément extérieure à laquelle la défense n'est pas pour et en elle-même possible. Dans des considérations plus prosaïques, il n'existe pas de dignité de la personne humaine après la mort parce que cette personne n'existe plus au sens juridique du terme. La question a pourtant trouvé un écho devant la Cour européenne des droits de l'homme qui a été conduite à examiner la possibilité d'appliquer l'article 3 de la Convention, interdisant les traitements inhumains et dégradants, à une mutilation de cadavres. En l'espèce ${ }^{68}$, les requérants ont saisi la cour à propos du meurtre de leur frère et de leur fils respectifs tués par des forces de sécurité pour des motifs politiques lors d'une fusillade. À la fin de celle-ci, les forces de sécurité se sont adonnées à de nombreuses mutilations sur les deux corps qui furent remis en morceaux à leurs familles. Appréciant de manière stricte la notion de traitement inhumain et dégradant, entendue comme constitutif d'une humiliation ou une souffrance se situant au-delà d'une action légitime sur un individu, la cour considère «que la qualité d'être humain s'éteint au décès et que, de ce fait, la prohibition des mauvais traitements ne s'applique plus à des cadavres [...] malgré la cruauté des faits en question $^{69}{ }$. Ainsi, nonobstant l'existence d'une opinion dissidente en l'espèce ${ }^{70}$, la dignité humaine ne peut donc s'apprécier qu'au regard de la qualité même d'espèce humaine intégrée à la communauté des vivants. Il s'agit donc de comprendre maintenant comment peut se poursuivre un processus de restitution de restes humains et dans quelle mesure, si la dignité humaine ne peut, en l'espèce, s'appliquer, si le traitement dans la dignité de l'article 16-1-1 permet de l'engager, lorsqu'une demande est formulée en ce sens.

68. CEDH, 27 février 2007, Akpinar et Altun c/. Turquie, $\mathrm{n}^{0}$ 56760/oo.

69. Ibid., point 82 .

70. Voir l'opinion partiellement dissidente de la juge Fura-Sandström, point 9: «Je regrette de ne pas avoir pu convaincre [...] que l'obligation de respecter la dignité et l'intégrité physique d'une personne demeure après le décès. » 


\subsubsection{L'érosion du principe d'inaliénabilité du domaine public}

En constatant diverses formes de restitutions aux États-Unis, en Angleterre ou en Australie, le Comité d'éthique met en avant la position conservatrice française qui se réfugierait « derrière l'argument juridique de l'inaliénabilité des collections ${ }^{71}$ ». La loi ${ }^{72}$ de 2002 relative aux musées de France réaffirme ce principe pour les objets affectés au domaine public dans cette catégorie d'établissements, en l'assortissant d'une exception, permettant un déclassement après avis d'une commission scientifique. Ainsi, s'il est vrai que l'édit de Moulins de 1566 pose un premier cadre de protection de la domanialité publique, la loi de 2002 opère subtilement une exception notable sous le prétexte de renforcer l'inaliénabilité en posant juridiquement son principe. En effet, même si l'on peut affirmer qu'antérieurement à ce cadre légal, les collections étaient sous la protection de leurs conservateurs, cette institutionnalisation du déclassement d'objets des collections publiques permet tout même, par une procédure, de constater si l'objet en cause remplit effectivement sa mission de service public. De plus, le rapport Rigaud remis au ministre chargé de la Culture en janvier 2008 s'interroge pour sa plus grande partie sur la pertinence des dispositions de 2002, tout en ne souhaitant pas une aliénation car « les quelques avantages financiers que les musées pourraient tirer de la vente d'œuvres me paraissent dérisoires par rapport à l'effet déplorable qui en résulterait ${ }^{73} »$. Notons toutefois que ces dispositions ont été vivement contestées par le comité "Patrimoine et Résistance » qui considère que cette loi «inscrit une première brèche dans la règle séculaire de l'inaliénabilité des collections du patrimoine national ${ }^{74}$ ». Le déclassement est rendu possible sur proposition de l'institution muséale, à partir de l'avis rendu par la Commission scientifique nationale des collections des musées de France ${ }^{75}$, créé par la loi de 2002 et qui

71. Jean-Claude Ameisen, Pierre Le Coz, op. cit., p. 10.

72. Loi $\mathrm{n}^{\circ}$ 2002-5, 4 janvier 2002 relative aux musées de France, JORF, 5 janvier 2002, p. 305.

73. Jacques Rigaud, Réflexion sur la possibilité pour les opérateurs publics d'aliéner des ceuvres de leurs collections, rapport remis au ministre de la Culture et la Communication le 20 janvier 2008, p. 48.

74. Jean Mennecier, «Introduction», Défense du patrimoine, bulletin du comité Patrimoine et Résistance, $\mathrm{n}^{0}$ 9, novembre 2009, p. 3.

75. Sur cette question, l'art. 11 de la loi «musées de France » a été codifié à l'art. L 451-5 du Code du patrimoine (ci-après « $\mathrm{CP}$ ») qui pose pour principe que: «Les biens constituant les collections des musées de France appartenant à une personne publique font partie de leur domaine public et sont, à ce titre, inaliénables [...]»; la saisine de la Commission est donc l'exception. 
disposait de cette compétence jusqu'à la loi ${ }^{76}$ du 18 mai 2010. Si cette commission n'a pas été supprimée par la loi de 2010, cette dernière a créé une Commission scientifique nationale des collections ${ }^{77}$, reprenant la compétence du déclassement, à celle qui se nomme désormais la Commission scientifique nationale des musées de France. La nouvelle Commission créé par la loi de 2010, est composée de quatre collèges qui se réunissent en fonction de l'institution dans laquelle se trouve le bien culturel faisant l'objet d'une demande de déclassement. La Commission créé par la loi de 2002 est, quant à elle, maintenue dans ses attributions précédentes ${ }^{78}$ mais n'aura donc géré qu'une seule situation de déclassement ${ }^{79}$ à l'occasion de la restitution par la France à l'Égypte de cinq fragments de peinture murale conservés au musée du Louvre et provenant du tombeau de Tetiky, un dignitaire de la XVIII ${ }^{\mathrm{e}}$ dynastie. En cela, la nouvelle Commission vient compléter le dispositif juridique car elle permet l'examen du déclassement de biens culturels du domaine public ne faisant pas partie des collections des musées de France. Si, comme nous l'avons vu, les corps font l'objet d'une sanctuarisation dans l'espace privé, où l'on encadre sa finalité par la crémation ou l'inhumation, le juge administratif a reconnu ${ }^{80}$ l'appartenance au domaine public des restes humains contenus dans les musées. Le principe du traitement respectueux, souhaité par l'article 16-1-1, n'entrave pas l'étude des restes humains au sein des musées et comme le rappelle le rapporteur public, la tradition du droit français du patrimoine est, qu'on le déplore ou non, "principalement un droit de la conservation et, exceptionnellement, un droit de l'échange ${ }^{81}$ ». Ainsi, seule l'adoption en 2010, d'une loi de validation, a permis une résolution d'un processus en faveur de la restitution de l'ensemble des têtes maories présentes dans les collections

76. Loi $\mathrm{n}^{0} 2010-501$ du 18 mai 2010 visant à autoriser la restitution par la France des têtes maories à la Nouvelle-Zélande et relative à la gestion des collections, JORF $\mathrm{n}^{\mathrm{o}} 0114 \mathrm{du} 19$ mai 2010, p. 9210.

77. Ses fonctions et attributions sont organisées par le décret $n^{0}$ 2011-160 du 08 février 2011 qui a été codifié par le décret $\mathrm{n}^{\mathrm{O}}$ 2011-573 du 24 mai 2011 aux articles R 115-1 à $\mathrm{R} 115-4$ du CP.

78. L'article R 451-3 du Code du patrimoine rappelle que la Commission créée en 2002 émet un avis sur les acquisitions faites dans les musées de France et se prononce préalablement au Haut Conseil sur l'appellation «musée de France» pour une institution qui en fait la demande.

79. Arrêté du 5 novembre 2009 portant déclassement de biens des collections des musées de France appartenant à l'État, JORF du 10 novembre 2009, p. 19457.

80. CAA Douai, 24 juillet 2008, Ville de Rouen, $\mathrm{n}^{0}$ 08DA00405.

81. Jacques Lepers, "Un reste humain peut-il appartenir au domaine public? », AJDA, 2008, p. 1896. 
des musées de France. Elle n'a pas pour autant résolu tous les problèmes, et la restitution des restes humains conservés dans le domaine public se retrouve encore confrontée à une difficulté majeure. En effet, comme le prévoit l'article L 451-7 du code du patrimoine «les biens incorporés dans les collections publiques par dons et legs ou, pour les collections ne relevant pas de l'État, ceux qui ont été acquis avec l'aide de l'État ne peuvent être déclassés ». Ainsi, il appartiendra à la nouvelle Commission scientifique « de confronter l'intérêt de conservation et d'exposition avec la dignité attachée aux restes humains, concrétisée par la demande d'un peuple "autochtone ${ }^{82 " ~ ", ~ m a i s ~ e ́ g a l e m e n t ~ s u r ~ l a ~ q u e s t i o n ~ d e ~ m a i n t e n i r ~}$ une inaliénabilité absolue sur la question spécifique des restes humains en tant que biens culturels présents dans les collections du domaine public par le biais de legs ou de dons. Il s'agit de s'emparer réellement de ce sujet qui en France n'a été traité qu'à partir de cas particuliers et symboliques et en ayant conscience que la plupart des restes humains contenus dans les collections muséales proviennent de dons ou de legs, du fait de leur nature même. Plusieurs solutions sont déjà envisageables telles que la réalisation d'un inventaire ${ }^{83}$ comme le prescrit la loi musée de France, mais étendu à l'ensemble des institutions publiques. Ce dernier aurait pour objectif une identification de ces restes humains avec l'appui de recherches historiques et pourraient ensuite faire l'objet d'une réclamation. En, effet, si une identification précise est avérée, l'information peut être communiquée sur le site internet de l'institution ou sur un site internet spécifique recensant l'ensemble des restes humains identifiés gérés par le ministère des affaires étrangères en lien avec les ambassades. Cette initiative permettrait une mise en conformité des engagements pris sous réserve par la France en vertu de la déclaration des Nations unies sur les droits des peuples autochtones qui dans ses articles $^{84}$ 11-2 et 12-1 prévoit une obligation pour les États d'assurer des mécanismes de réparation et donc un droit au retour des restes humains

82. Xavier Bioy, «Le statut des restes humains archéologiques », Revue de droit public, t. $127-2011,1$, p. 108.

83. Article L 451-2 CP: «Les collections des musées de France font l'objet d'une inscription sur un inventaire. Il est procédé à leur récolement tous les dix ans ».

84. Déclaration des Nations unies sur les droits des peuples autochtones du 13 septembre 2007, article 11-2: "Les États doivent accorder réparation par le biais de mécanismes efficaces - qui peuvent comprendre la restitution - mis au point en concertation avec les peuples autochtones, en ce qui concerne les biens culturels, intellectuels, religieux et spirituels qui leur ont été pris sans leur consentement préalable, donné librement et en connaissance de cause, ou en violation de leurs lois, traditions et coutumes. » et article 12-1: «Les peuples autochtones ont le droit [...] au rapatriement de leurs restes humains. » 
à leur terre d'origine. Encore faudrait-il pouvoir surmonter l'obstacle de l'article L 451-7 du Code du patrimoine et en la matière, une solution législative s'impose, qui pourrait conduire à ce que le "matériel culturel sensible ${ }^{85}$ » ayant été acquis par dons ou legs, puisse également être déclassé, par exemple, sur avis conforme de l'institution gardienne et de la Commission scientifique nationale des collections.

\subsection{La diversité des approches de restitution face aux nécessités d'un dialogue interculturel}

Si cette question de restitution de restes humains n'a trouvé en France qu'une réponse partielle et casuistique, une étude comparative permet de mettre en avant les réponses globales qu'ont pu apporter certains États sur ce sujet (2.2.1). Ainsi, cette confrontation de points de vue permet d'avancer des solutions avec un regard différent, au service d'un dialogue interculturel (2.2.2).

\subsubsection{Des solutions comparatives codificatrices}

En matière de restitution, la pratique internationale est extrêmement diversifiée et des démarches de codification ont pu voir le jour comme ce fut le cas, en premier lieu, aux États-Unis avec le Native American Graves Protection and Repatriation Act ${ }^{86}$ (NGAPRA) qui organise un inventaire dans les musées ainsi que la possibilité pour les tribus indiennes natives de demander le retour de leurs restes humains. Le NGAPRA impose aux communautés indiennes la preuve de leur affiliation culturelle dans le cadre d'une demande visant la restitution d'un objet sacré, qu'il soit funéraire ou non, mais considéré comme faisant partie de leur patrimoine culturel. Un contentieux important a vu le jour et l'une des affaires les plus emblématiques a été celle de l'homme de Kennewick, réclamé par plusieurs tribus indiennes, alors que son squelette avait été découvert dans une rivière. Les scientifiques estimaient qu'il remontait

85. Expression utilisée par le Conseil international des musées (ICOM) dans son code de déontologie aux articles 2.5 : «les collections composées de restes humains ou d'objets sacrés ne seront acquises qu'à condition de pouvoir être conservées en sécurité et traitées avec respect. Cela doit être fait en accord avec les normes professionnelles et, lorsqu'ils sont connus, les intérêts et croyances de la communauté ou des groupes ethniques ou religieux d'origine »; 3.7 (missions de recherche); et 4.3 (exposition des objets sensibles).

86. Loi du 16 novembre 1990, disponible en ligne à l'adresse http://www.nps.gov/nagpra/mandates/25usc3001etseq.html (consulté le 27 janvier 2013). 
à une période bien antérieure à l'arrivée des amérindiens, son apparence étant caucasienne. Il n'a finalement pas été restitué car il apparaissait notamment comme "un maillon important dans la preuve de cette théorie, offrant ainsi une meilleure compréhension de l'histoire de l'évolution de l'homme ${ }^{87}$ ». Alors que l'exemple de la législation des États-Unis mériterait un plus long développement ${ }^{88}$, il s'avère plus pertinent pour notre étude d'utiliser des points de comparaison avec un pays qui a pratiqué, comme la France, à l'échelle mondiale une forme de colonisation, c'est-à-dire le Royaume-Uni. Sa pratique muséale considère que ce n'est pas à l'État de gérer le bien public mais que celui-ci doit pouvoir être organisé par des trustees c'est-à-dire des groupes de personnes qui, de par leur jugement intellectuel et moral, gèrent les biens et la politique du musée au service du public. Les trustees sont les détenteurs légaux de la propriété, sans pouvoir toutefois en bénéficier. À titre de comparaison, on peut de par leur forme, les rapprocher du système fiduciaire: ils gèrent en tant qu'institution tierce, au nom du public, un patrimoine constitué au sein d'un musée. La réflexion sur les restitutions au Royaume-Uni a été un travail de longue haleine puisqu'elle a débuté dès les années 1980, lorsque l'University College of London rendit pour la première fois une tête maorie, en 1987. Pour prendre un autre exemple concernant cette question, nous pouvons nous pencher sur l'histoire ${ }^{89}$ de Yagan, un jeune aborigène de la communauté Noongah, décapité en 1833 et dont la dépouille fut emmenée en Angleterre par le lieutenant Robert Dale, pour être entreposée dans les collections de la Royal Institution puis au Liverpool City Museum, jusqu'en 1964. Cette dernière institution ayant rencontré des problèmes de conservation, elle l'inhuma dans le cimetière d'Everton. Il fut redécouvert en 1993, puis restitué par Liverpool à une délégation aborigène en 1997, pour laquelle ce n'est " pas seulement une nécessité spirituelle mais aussi un élément essentiel dans la préservation de leur culture et de leur mode de vie ${ }^{90} »$. Les restitutions pendant cette période, ainsi que les débats, se poursuivirent

87. Wendy Crowther, «How Kennewick man uncovered the problems in NGAPRA », Journal of Land, Resources and Environmental Law, vol. 20, 2000, p. 280: « .. a significant link in proving in this theory, thus providing a greater understanding of human evolutionary history..."

88. Zoe E. Niesel, "Better late than never? The effect of the NAGPRA's 2010 regulations", Wake Forest Law Review, vol. 46, 2011, p. 837-865.

89. Racontée par Kevin Chamberlain, "We need to lay our ancestors to rest- the repatriation of indigenous human remain and the human rights act ", Art, Antiquity and Law, vol. 10, $\mathrm{n}^{\circ}$ 4, décembre 2005, p. 329. 
avec l'installation, en 2001, par le ministre de la Culture, Alan Howarth, d'un groupe de travail sur les restes humains, lequel rendit son rapport en novembre 2003. À partir de l'enquête réalisée notamment sur la base de questionnaires envoyés aux institutions muséales, il s'est avéré que sur les 146 musées qui ont répondu, 132 ont des collections avoisinant les 61000 pièces. Les propositions du groupe de travail ont abouti à l'adoption de deux types d'actes: un guide pour l'éthique des restes humains dans les musées et la section 47 de l'Human Tissue Act, refondu pour l'occasion en 2004 et entré en vigueur le 6 octobre 2005. Cette loi a été adoptée pour permettre aux trustees de disposer d'un large champ d'action, puisque tous ceux qui sont énoncés peuvent "transférer de leurs collections n'importe quel reste humain d'une personne décédée de moins de 1000 ans avant la date d'entrée en vigueur du présent article si cela leur apparaît approprié de le faire pour une raison quelconque en relation ou non avec leurs autres fonctions ${ }^{91} »$. Cette limite temporelle de 1 ooo ans peut apparaître comme arbitraire, mais elle est le signe de la volonté pour les autorités britanniques de clore le débat autour de la restitution de restes humains considérés comme des éléments du patrimoine d'étude archéologique. Il s'agissait de réfléchir à la question dans un cadre global en traitant également des revendications culturelles car, dans ce cas, «les demandes doivent être traitées comme un dialogue ouvert et constructif entre le musée et les demandeurs ${ }^{92}$ ». Ainsi, le guide de bonnes pratiques vient préciser la pensée du législateur sur la limitation temporelle, en découpant le champ d'action des trustees en trois différentes étapes, partant du constat que «la grande majorité des demandes qui ont été faites pour le retour ont concerné les restes de personnes à l'étranger qui sont morts dans une période comprise entre les 100 et 300 dernières années. Cela correspond plus étroitement à la période de l'expansion des empires coloniaux européens avec ses effets subséquents sur les peuples autochtones, une période qui ne remonte pas

90. Kevin Chamberlain, art. cité, p. $330:$ «... not only a spiritual necessity but also an essential element in the preservation of their culture and way of life."

91. Human Tissue Act, 2004, section 47 , Power to de-accession human remains, $\$ 2: \ll \ldots$ may transfer from their collection any human remains which they reasonably believe to be remains of a person who died less than one thousand years before the day on which this section comes into force if it appears to them to be appropriate to do so for any reason, whether or not relating to their other functions. "

92. Guidance for the Care of Human Remains in Museums: "Requests should be dealt with as an open and constructive dialogue between the museum and the claimants", p. 24, disponible sur http://webarchive.nationalarchives.gov.uk/+/http://www.culture.gov.uk/images/publications/Guidar (consulté le 27 janvier 2013). 
à plus de $500 \mathrm{ans}^{93}$ ». Le Royaume-Uni a donc choisi de se placer, par la reconnaissance des faits coloniaux, comme le pays qui offre la possibilité tant à l'État d'origine, qu'aux descendants directs ou même à une communauté disposant d'un intérêt et/ou d'une continuité culturelle de venir demander la restitution de restes humains. En vue de la réalisation de ces objectifs, le British Museum, par le biais de ses trustees, a adopté un règlement intérieur ${ }^{94}$ le 6 octobre 2006, venant définir sa conduite politique en la matière. L'article 5.1 de ce règlement définit le cadre de travail des trustees et leurs objectifs, comme devant assurer les collections muséales pour le bien des générations présentes et futures, ce qui suppose de pouvoir gérer efficacement leur état de conservation, tant qu'une demande de restitution n'est pas formulée. Ainsi, le RoyaumeUni se distingue nettement de la France, où cette question n'a pas fait l'objet d'un traitement particulier lors de l'adoption en 2002 de la loi relative aux musées de France. Cependant, des lois ponctuelles sur des restes humains spécifiques telles que la Vénus hottentote ${ }^{95}$ ou les têtes maories par la loi du 18 mai 2010, ont été adoptées pour répondre aux exigences des gouvernements et communautés respectives et surmonter le problème de l'article L 451-7 du Code du patrimoine. Il s'agit maintenant d'analyser quelles pourraient être les solutions envisagées, en se demandant en premier lieu si la restitution doit devenir une fin en soi ou si des possibilités de dialogue sont envisageables.

\subsection{2. Écouter, traduire, choisir: trois exigences au service d'un dialogue interculturel}

En limitant fortement les possibilités de conservation et d'exposition de restes humains, le Comité d'éthique fait toutefois dans son avis plusieurs recommandations quant à leurs possibilités de restitution. Il précise que le «le pays d'origine qui a formulé la demande [...] est celui d'un peuple actuel ${ }^{96}$ » et que celle-ci doit se faire « en concertation

93. Ibid., p. 27: "The vast majority of claims that have been made for return have concerned the remains of overseas people who died within the last 100-300 years. This corresponds most closely to the period when expansion took place by European powers with its subsequent effect on Indigenous peoples-a period that does not go back further than 500 years."

94. The British Museum Policy on human remains, adopté le 6 octobre 2006, disponible sur http://www.britishmuseum.org/PDF/Human \protect|kern+.1667em|relax\%2oRemains \protect। (consulté le 27 janvier 2013).

95. Loi $\mathrm{n}^{\mathrm{O}} 2002-323$ du 6 mars 2002 relative à la restitution par la France de la dépouille mortelle de Saartjie Baartman à l'Afrique du Sud.

96. Jean-Claude Ameisen, Pierre Le Coz, op. cit., p. 12. 
avec l'ensemble des institutions des directeurs des institutions concernées ${ }^{97}$ ». Cependant, il n'assigne qu'une seule destination à ces restes humains en considérant que l'objectif du retour est de n'être ni « exposé ni conservé [...] mais inhumé ${ }^{98}$ ", ce qui détruit le sens même d’une restitution, entendu comme le «fait de remettre au propriétaire une chose dont il avait été privé indûment ou involontairement ${ }^{99}$ ». En effet, admettre la restitution implique le fait d'une dépossession matérielle de l'institution muséale. Celle-ci, pour des raisons légitimes, peut toutefois s'enquérir de la destination finale de ces restes humains. Si le principe d'une restitution au sens juridique est une rétribution sans condition du fait d'une dépossession originaire, celle-ci s'étant produite depuis une longue période, le principe de bonne foi peut être un élément d'appréciation pour ces institutions qui se sont retrouvées dépositaires de telles pièces. Ainsi, si ce qu'il advient ensuite des restes humains relève des communautés concernées, cela n'empêche pas pour autant la tenue d'un dialogue pour une conservation, du moins au sens immatériel, et non une disparition. Le musée Te Papa Tongarewa de Wellington dans le cadre de son programme de rapatriement essaie de concilier les exigences scientifiques et le devoir des peuples envers leurs morts. Ce programme, nommé Karanga Aotearoa ou l'appel de la terre natale a permis au musée de se doter d'un "Wahi Tapu, lieu sacré où l'on conserve les restes humains le temps de pouvoir les restituer à leur $\operatorname{tribu}^{100} »$. À ce jour une trentaine de têtes ont pu être formellement identifiées grâce à leurs tatouages et restituées à leur tribu d'origine. Il est probable que les têtes qui ne seront pas identifiées resteront à disposition des maoris qui réglementent strictement l'accès de ce lieu. Le musée essaye de rassembler toutes les informations nécessaires permettant de conserver une trace de cet héritage en ayant conscience de leur valeur pour la recherche. Le colloque organisé par le Muséum ${ }^{101}$ en leur présence, quelques jours avant la restitution officielle, est un exemple d'un dialogue interculturel autour duquel chacun s'apporte un aspect de la connaissance ignoré par l'autre, pour sa plus grande partie. Si des règles

97. Ibid., p. 13 .

98. Ibid.

99. Gérard Cornu (dir.), Vocabulaire juridique, $7^{\mathrm{e}}$ éd., Paris, PUF, 2005, p. 810.

100. Simon Jean, Le rôle des restes humains au sein de nos collections nationales: l'exemple des têtes maories, mémoire de master professionnel, université de Rouen, spécialisation patrimoine, année 2008-2009, p. 90.

101. Colloque scientifique sur la restitution des têtes maories du 20 janvier 2012 organisé à l'auditorium de la grande galerie de l'Évolution du Muséum national d'histoire naturelle (MNHN). 
juridiques concernant la restitution de restes humains sont nécessaires, il est encore plus indispensable qu'elles soient les plus souples possibles pour un sujet dans lequel «la logique du paradigme communautaire, visant le partage et la négociation permanente de consensus et visant la complémentarité des différences ${ }^{102}$ » est au centre d'un débat forcément interculturel. Au-delà de la difficulté du principe d'inaliénabilité absolue pour les biens faisant l'objet de dons et de legs, résolu partiellement et péniblement par des lois de circonstances sur la Vénus hottentote et les têtes maories, le débat doit également se poursuivre au sein des instances muséales. En revenant sur les problématiques liées à la conservation et l'exposition, si le consentement ne suffit pas à la tenue d'une telle exposition comme garantie de légitimation éthique, il faut contrôler le cadre dans lequel cette exposition peut avoir lieu. En effet, la mise en scène participe à la conscientisation du respect face à un reste humain et à cet égard, il serait souhaitable que les musées puissent se doter d'une charte ${ }^{103}$ de bonne gestion, régulant l'ensemble des pratiques autour de la conservation, de l'exposition et des politiques de restitution. L'issue pédagogique est toujours d'actualité : il ne faut pas fermer les yeux mais savoir appréhender la mort tant dans l'importance des collections que dans la rationalisation d'une pratique. En somme, il ne faut pas chercher le choc émotionnel sans pour autant se voiler la face. La dimension lucrative peut être contournée, comme l'a montré l'exemple de Clermont-Ferrand. Une visibilité historique sur les collections s'avère donc nécessaire, tout en soulignant les intérêts scientifiques derrière la conservation de restes humains. Il s'agit également d'ouvrir clairement un débat autour de la Déclaration des peuples autochtones en France, qui ne la considère pas applicable aux peuples ${ }^{104}$ constitués sur son territoire. Pour parer les craintes de ceux qui estimeraient que de tels débats reviendraient à ouvrir une boîte de Pandore visant à vider les collections muséales, ces affirmations doivent être contredites puisque tous les peuples ne réclament pas des restes humains. Dans un

102. Christoph Eberhard, Droits de l'homme et dialogue interculturel, Paris, Connaissances et Savoirs, 2011, p. 481.

103. L'ICOM (Conseil international des musées) a adopté le 16 août 2013 un Code d'éthique pour les Muséums d'histoire naturelle, dont la section I concerne les restes humains. Ce Code est sur http://icom.museum/uploads/media/nathcode_ethics_en_01.pdf (consulté le 27 janvier 2013).

104. Le Conseil constitutionnel ne reconnaît que l'unicité du peuple français: CC, décision n ${ }^{0}$ 91-29o DC du 9 mai 1991, loi portant statut de la collectivité territoriale de Corse, considérant 11 et 13 . 